\title{
Polynomial behavior of the Honda formal group law
}

\author{
Malkhaz Bakuradze ${ }^{1}$
}

Received: 10 June 2015 / Accepted: 25 February 2016 / Published online: 17 March 2016

(C) Tbilisi Centre for Mathematical Sciences 2016

\begin{abstract}
This note provides the calculation of the formal group law $F(x, y)$ in modulo $p$ Morava $K$-theory at prime $p$ and $s>1$ as an element in $K(s)^{*}[x][[y]]$ and some applications to relevant examples.
\end{abstract}

Keywords Formal group law $\cdot$ Morava $K$-theory

Mathematics Subject Classification 55N22 - 55N22

\section{Introduction}

Let $K(s)^{*}(-)$, be the $s$-th Morava $K$-theory at prime $p$ [4]. The coefficient ring $K(s)^{*}(p t)$ is the Laurent polynomial ring in one variable $\mathbb{F}_{p}\left[v_{s}, v_{s}^{-1}\right]$, where $\mathbb{F}_{p}$ is the field of $p$ elements and $\operatorname{deg}\left(v_{s}\right)=-2\left(p^{s}-1\right)$.

Let $F(x, y)$ be the formal group law in $K(s)^{*}(-)$ theory [5]. The purpose of this note is to prove that if $s>1$, the formal group law $F(x, y)$ is a polynomial modulo $y^{p^{i(s-1)}}$ (or equivalently modulo $x^{p^{i(s-1)}}$ ) for any $i \geq 1$, see Theorem 2.1. This fact was never mentioned before in the literature even though the proof is quite simple. We also want to have a method for explicit calculation. The idea is to apply the Ravenel

Communicated by Vladimir Vershinin.

The author was supported by Volkswagen Foundation, Ref.: I/84 328.

$凶 \quad$ Malkhaz Bakuradze

malkhaz.bakuradze@tsu.ge

1 Faculty of exact and natural sciences, A.Razmadze Math. Institute of Iv. Javakhishvili Tbilisi State University, Tbilisi, Georgia 
formula [8] involving Witt's symmetric polynomials. The proof does not work for $s=1$. The particular case (2.3) of Theorem 2.1 was applied in several papers by the author, also [10-12], and [6]. Some other motivation is given in Sect. 3.

\section{The statement}

Recall the recursive formula from Ravenel's green book, (see [8], 4.3.8) for the formal group law. In $K(s)^{*}(-)$ theory it reads (we set $v_{s}=1$ and $q=p^{s-1}$ )

$$
F(x, y)=F\left(x+y, w_{1}(x, y)^{q}, w_{2}(x, y)^{q^{2}}, w_{3}(x, y)^{q^{3}}, \ldots\right)
$$

where $F(x, y, z, \ldots)=x \oplus_{F} y \oplus_{F} z \oplus_{F} \cdots$ is the iterated $x \oplus_{F} y=F(x, y)$ and $w_{j}$ are $\bmod (p)$ Witt's integral symmetric homogeneous polynomials of degree $p^{j}$ :

$$
x^{p^{n}}+y^{p^{n}}=\sum_{j} p^{j} w_{j}(x, y)^{p^{n-j}} .
$$

In particular

$$
\begin{aligned}
& w_{0}=x+y, \\
& w_{1}=-\sum_{0<j<p} p^{-1}\left(\begin{array}{l}
p \\
j
\end{array}\right) x^{j} y^{p-j} .
\end{aligned}
$$

We will need that $\operatorname{deg}\left(w_{j}\right)=p^{j}$ and that $w_{j}(x, 0)=w_{j}(0, y)=0$, for $j>0$.

Clearly we have

$$
F(x, y)=x+y \text { modulo } y^{q} .
$$

One has for $s>1$ (see [1])

$$
F(x, y)=x+y+w_{1}(x, y)^{q} \text { modulo } y^{q^{2}} .
$$

We now want to prove that for $s>1, F(x, y)$ is again a polynomial modulo $y^{q^{n}}$ for any $n$. The idea is to apply (2.1).

Theorem 2.1 One has $F(x, y) \in K(s)^{*}[x][[y]]$ for the formal group law $F(x, y)$ in $\bmod p$ Morava $K(s)^{*}(-)$ theory at $p$ and $s>1$.

A method for calculation of $F(x, y)$ modulo $y^{q^{n}}$ is given by the Ravenel formula (2.1) and induction on $n$.

Proof By induction hypothesis, we have that $F(x, y)$ modulo $y^{q^{k}}, k \leq n$ is a polynomial, say $P_{k}(x, y)$. By (2.2) and (2.3) we have

$$
P_{1}(x, y)=x+y, \quad P_{2}(x, y)=x+y+w_{1}(x, y)^{q} .
$$


Induction step: Let us work modulo $y^{q^{n+1}}$. Then (2.1) implies

$$
F(x, y) \equiv F\left(x+y, w_{1}(x, y)^{q}, w_{2}(x, y)^{q^{2}}, \ldots, w_{n}(x, y)^{q^{n}}\right) .
$$

By induction hypothesis we have

$$
\begin{aligned}
F(x, y) & \equiv z_{1}+w_{n}^{q^{n}}, \quad \text { where } z_{1}=F\left(x+y, w_{1}^{q}, \ldots, w_{n-1}^{q^{n-1}}\right) \\
z_{1} & \equiv P_{2}\left(z_{2}, w_{n-1}^{q^{n-1}}\right), \quad z_{2}=F\left(x+y, w_{1}^{q}, \ldots, w_{n-2}^{q^{n-2}}\right) \\
z_{2} & \equiv P_{3}\left(z_{3}, w_{n-2}^{q^{n-2}}\right), \quad z_{3}=F\left(x+y, w_{1}^{q}, \ldots, w_{n-3}^{q^{n-3}}\right) ; \\
& \ldots \\
z_{n-2} & \equiv P_{n-1}\left(z_{n-1}, w_{2}^{q^{2}}\right), \quad z_{n-1}=F\left(x+y, w_{1}^{q}\right) ; \\
z_{n-1} & \equiv P_{n}\left(x+y, w_{1}^{q}\right) .
\end{aligned}
$$

Accordingly $F(x, y)$ is again a polynomial modulo $y^{q^{n+1}}$ for any natural $n$. Therefore one can collect the coefficients at $y^{j}, j<q^{n+1}$ for any $n$ and write

$$
F(x, y)=\sum A_{l}(x) y^{l} \in K(s)^{*}[x][[y]] .
$$

The proof above gives more, namely one can evaluate the degree of the polynomial $A_{l}(x)$.

Proposition 2.2 In $F(x, y)=\sum \alpha_{i j} x^{i} y^{j}$ we have $\alpha_{i j}=0$ for $i>(p q)^{n}$ whenever $j<q^{n}$.

Proof Base case is obvious. Induction step: by Theorem 2.1 we have modulo $y^{q^{n+1}}$

$$
F(x, y) \equiv P_{n}(x+y, z)+w_{n}(x, y)^{q^{n}}, \quad z=F\left(w_{1}(x, y)^{q}, \ldots, w_{n-1}(x, y)^{q^{n-1}(\mathcal{L} .5)}\right.
$$

The term $w_{n}^{q^{n}}$ is of degree $(p q)^{n}$ hence is irrelevant.

Let $\beta_{i j}(x+y)^{i} z^{j}$ be any term of the polynomial $P_{n}(x+y, z)$. By induction hypothesis we have

$$
i \leq(p q)^{n} \quad \text { whenever } j<q^{n}
$$

Then $z^{j}$ is a polynomial in $w_{1}^{q}, \ldots, w_{n-1}^{q^{n-1}}$. Therefore it has the terms

$$
\left(w_{1}^{q}\right)^{j_{1}} \cdots\left(w_{n-1}^{q^{n-1}}\right)^{j_{n-1}}, \quad \text { with } j_{1}<q^{n}, \ldots j_{n-1}<q^{2}
$$

as we work modulo $y^{q^{n+1}}$. 
Therefore $z^{j}$, as a polynomial in $x$ and $y$, has the terms of total degree

$p q j_{1}+(p q)^{2} j_{2}+\cdots+(p q)^{n-1} j_{n-1}<p q^{n+1}+p^{2} q^{n+1}+\cdots+p^{n-1} q^{n+1}$.

Thus for any term of $P_{n}(x+y, z)$

$$
\text { the total degree }<(p q)^{n}+q^{n+1} \sum_{1 \leq l \leq n-1} p^{l}<q^{n+1} \sum_{1 \leq l \leq n} p^{l}<(p q)^{n+1} \text {. }
$$

This completes the proof.

\section{Some simple applications}

The particular case $n=2$ of Theorem 2.1 was already applied in several papers.

Consider an extension of $C_{p^{k}}$ by an elementary abelian $p$-group. That is $G$ fits into an extension

$$
1 \rightarrow\left(C_{p}\right)^{l} \rightarrow G \rightarrow C_{p^{k}} \rightarrow 1
$$

It is known [7,13] that $G$ is good, i.e., $K(s)^{*}(B G)$ is generated by Chern classes. However the explicit account of the ring structure was never done for $k>1$.

The examples for the case $k=1$ was considered in [2,3]. Namely let $\xi$ be a complex $m$-plane bundle over the total space of a cyclic covering $\pi: X \rightarrow X / C_{p}$ of prime index $p$. Let $c$ be the Chern class of $X \times_{C_{p}} \mathbb{C} \rightarrow X / C_{p}$, the complex line bundle associated to covering $\pi$. In [1] we showed that modulo image of the transfer homomorphism the $i$-th Chern class $c_{i}$ of the transferred bundle $\xi$ can be written as a polynomial $\mathcal{A}_{i}$ in Chern classes $c_{p}, c_{2 p}, \cdots c_{m p}$ and $c^{p-1}$. Using the polynomials $\mathcal{A}_{i}$ in [2,3], we showed for various examples of finite groups that $K(s)^{*}(B G)$ is the quotient of a polynomial ring by an ideal for which we listed explicit generators.

We recall that Morava $K$-theory for a cyclic group is the truncated polynomials [9]. In particular

$$
K(s)^{*}\left(B C_{p^{k}}\right)=\mathbb{F}_{p}\left[v_{s}, v_{s}^{-1}\right][u] / u^{p^{k s}} .
$$

Also

$$
K(s)^{*}(B U(m))=\mathbb{F}_{p}\left[v_{s}, v_{s}^{-1}\right]\left[\left[c_{1}, \ldots, c_{m}\right]\right]
$$

and because of the Künneth isomorphisms

$$
K(s)^{*}\left(B U(m) \times B C_{p^{k}}\right)=K(s)^{*}(B U(m)) \otimes K(s)^{*}\left(B C_{p^{k}}\right) .
$$

Theorem 2.1 enables to write explicitly the relations derived by formal group law and splitting principle as relations in Chern classes of complex representations. 
In particular, let $\theta$ be the line complex bundle over $B G$, associated to covering $\pi: B H \rightarrow B G, H=\left(C_{p}\right)^{l}, \eta$ is the pullback by projection on the first factor $H \rightarrow C_{p}$ of the canonical bundle over $B C_{p}$ and $\pi ! \eta$ is the transferred $\eta$. Then we have the bundle relation over $B G$

$$
\pi ! \eta \otimes \theta=\pi ! \eta
$$

The relation (3.1) holds because of Frobenius reciprocity of the transfer homomorphism of covering $\pi$ in complex $K$-theory:

$$
\pi ! \eta \otimes \theta=\pi_{!}\left(\eta \otimes \pi^{*}(\theta)\right)=\pi_{!}(\eta \otimes 1)=\pi ! \eta .
$$

This implies the relations

$$
c_{i}(\pi ! \eta \otimes \theta)=c_{i}(\pi ! \eta)
$$

in $K(s)^{*}(B G)$. If we want to write everything in the explicit form, we have to apply the splitting principle to (3.1) and write formally $\pi ! \eta$ as the sum of line bundles $\pi ! \eta=\eta_{1}+\cdots+\eta_{p^{k}}$. Thus we have

$$
\eta_{1}+\cdots+\eta_{p^{k}}=\eta_{1} \otimes \theta+\cdots+\eta_{p^{k}} \otimes \theta
$$

Using the elementary symmetric polynomials $\sigma_{i}, i=1, \ldots, p^{k}$ we can write

$$
c_{i}(\pi ! \eta)=\sigma_{i}\left(c_{1}\left(\eta_{1}\right), \ldots, c_{1}\left(\eta_{p^{k}}\right)\right) .
$$

In fact we have the following equations

$$
\sigma_{i}\left(c_{1}\left(\eta_{1}\right), \ldots, c_{1}\left(\eta_{p^{k}}\right)\right)=\sigma_{i}\left(F\left(c_{1}\left(\eta_{1}\right), c_{1}(\theta)\right), \ldots, F\left(c_{1}\left(\eta_{p^{k}}\right), c_{1}(\theta)\right)\right) .
$$

To rewrite (3.2) explicitly, we apply Theorem 2.1 for each term $F\left(c_{1}\left(\eta_{j}\right), c_{1}(\theta)\right)$ and write it as a polynomial in $c_{1}\left(\eta_{j}\right)$ and $u=c_{1}(\theta)$ as $\theta^{p^{k}}=1$ implies $u^{p^{k s}}=0$. This is because $c_{1}\left(\theta^{p^{k}}\right)=\left[p^{k}\right]\left(c_{1}(\theta)\right)$ and $[p](x)=x^{p^{s}}$ for the Honda formal group law.

Finally we turn to the Chern classes. In this way one can try to compute $K(s)^{*}(B G)$ as a quotient of a polynomial ring (as $G$ is finite) by relations ideal. For this we have to establish two facts: the classes we define generate, and the list of relations is complete. To check the latter is easier if the relations are given as explicit polynomials.

Acknowledgements The author would like to thank the editors and the referee for several helpful comments.

\section{References}

1. Bakuradze, M., Priddy, S.: Transferred Chern classes in Morava $K$-theory. Proc. Am. Math. Soc. 132, 1855-1860 (2004) 
2. Bakuradze, M., Jibladze, M.: Morava K-theory rings of groups $G_{38}, G_{41}$ of order 32 . J K-Theory 13, 171-198 (2014)

3. Bakuradze, M.: Morava $K$-theory rings for the modular groups in Chern classes. K-theory 38(2), 87-94 (2008)

4. Johnson, D.C., Wilson, W.S.: B P operations and Morava's extraordinary $K$-theories. Math. Z. 144(1), 55-75 (1975)

5. Honda, T.: On the theory of commutative formal groups. J. Math. Soc. Jpn. 22(2), 213-246 (1970)

6. Kitchloo, N., Laures, G., Stephen Wilsona, W.: Morava $K$-theory of spaces related to BO. Adv. Math. 189, 192-236 (2004)

7. Kriz, I.: Morava $K$-theory of classifying spaces: some calculations. Topology 36, 1247-1273 (1997)

8. Ravenel, D.C.: Complex Cobordism and Stable Homotopy Groups of Spheres. Academic Press, Cambridge (1986)

9. Ravenel, D.C.: Morava $K$-theories and finite groups. Contemp. Math. 12, 289-292 (1982)

10. Schuster, B.: Morava $K$-theory of groups of order 32. Algebraic Geom. Topol. 11, 503-521 (2011)

11. Schuster, B.: $K(n)$ Chern approximations of some finite groups. Algebraic Geom. Topol. 12(3), 16951720 (2012)

12. Schuster, B.: Morava $K$-theory of classifying spaces, Habilitationsschrift, pp. 124 (2006)

13. Yagita, N.: Note on BP-theory for extensions of cyclic groups by elementary abelian $p$-groups. Kodai Math. J. 20(2), 79-84 (1997) 